\title{
Detection of Bacteroides fragilis and Bacteroides melaninogenicus by direct immunofluorescence
}

\author{
M LABBE, N DELAMARE, F PEPERSACK, F CROKAERT, AND \\ E YOURASSOWSKY
}

From the Hôpital Universitaire Brugmann, Service de Biologie Clinique, Brussels, Belgium

SUMmARY A new diagnostic kit, which contains a polyvalent antiserum for either Bacteroides fragilis or Bacteroides melaninogenicus, was tested for reliability and specificity on 146 clinical samples of different origin. A correlation between the culture and immunofluorescence was observed for $B$. fragilis in $87.39 \%$ of cases and for B. melaninogenicus in $81.48 \%$ of cases. When pure cultures were tested, aerobically as well as anaerobically, false-positive reactions were observed with staphylococci and Clostridium ramosum spores. The well-defined morphology of these bacteria and spores allows for the elimination of any diagnostic error. The method is rapid, and the margin for error is limited. The test gives a semiquantitative idea of the number of bacteroides organisms present in the clinical specimens even in the presence of a mixed flora.

Bacteroides from the fragilis and melaninogenicus groups are the anaerobic bacteria often isolated most frequently from various wounds. ${ }^{1-4}$

These organisms are not easily isolated from culture material. This is due to various factors such as poor technique for obtaining the specimens, poor transport, or incorrect culture methods. In addition, it is possible to have facultative aerobic organisms inhibiting the growth of anaerobic ones. These factors tend to reduce the diagnostic accuracy of a clinical evaluation based solely on an anaerobic culture. ${ }^{1}$ The severity of clinical infections caused by $B$. fragilis and their resistance to penicillins, cephalosporins, and aminoglycosides make an early and accurate diagnosis necessary. Immunofluorescence has been studied by various investigators in order to obtain a rapid diagnosis. ${ }^{5-8}$

Our study compared two techniques for detecting these anaerobic bacteria, that is, the traditional culture method and two new kits, Fluoretec $F$ and $M^{*}$. The kits contained fluorescein-labelled polyvalent antisera to either the $B$. fragilis or Bacteroides melaninogenicus groups. In addition, there was a rhodamine counterstain, which markedly decreased the background fluorescence from protein A-con-

\footnotetext{
* Registered trademark, Pfizer Diagnostics.

Received for publication 18 February 1980
}

taining material. The kits and culture methods were used to evaluate laboratory stock cultures and fresh clinical material such as pus and other wound exudates.

\section{Material and methods}

SAMPLES

Sixty laboratory stock cultures were tested with the Fluoretec kits. These stock cultures were obtained from various clinical sources, identified by the method of Holdeman et al. ${ }^{9}$ and stored in liquid nitrogen. Three reference strains were also tested (Table 1).

One hundred and nineteen clinical specimens were examined by immunofluorescence and the usual anaerobic bacteriological methods. These specimens were from various sources: deep ulcers (47), peritoneal fluid (15), abdominal and other wounds (22), blister fluid (13), abdominal abscesses (5), blood cultures (6), and exudates from various sources (11). The modes of transportation used were swabs (70) (Culturette-Marion Scientific Corp), syringes (32) (Plastipak BD), blood culture bottles (61) (Evac Brucella Broth, Pfizer), and sterile tubes (11). All of the specimens were examined with the $B$. fragilis antiserum. Twenty-seven specimens gave a red fluorescence when examined under ultraviolet light. These specimens were then tested with the B. melaninogenicus antiserum. 
Table 1 Immunofluorescence of different microbial strains with specific antiserum for Bacteroides fragilis and Bacteroides melaninogenicus

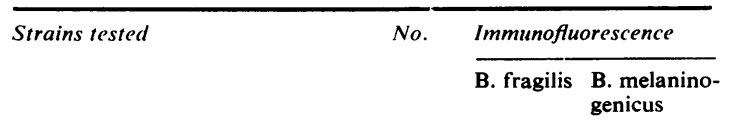

\section{Aerobes}

Haemophilus influenzae

Escherichia coli

Salmonella enteritidis

Proteus mirabilis

Pseudomonas aeruginosa

Erwinia herbicola

Klebsiella pneumoniae

Pasteurella multocida

Providencia stuartii

Staphylococcus aureus

Staphylococcus albus

Corynebacterium sp.

Streptococcus faecalis

Anaerobes

$C$. ramosum spores

Bacteroides putridinis

Bacteroides bivius

Bacteroides melaninogenicus

Bacteroides oralis

Bacteroides ureolytycus

Bacteroides ochraceus

Bacteroides fragilis NTCC 9343

Bacteroides thetaiotaomicron

Bacteroides distasonis ATCC 8503

Bacteroides vulgatus ATCC 8482

Bacteroides uniformis

Bacteroides ovatus

Peptococcus sp.

Peptostreptococcus sp.

Fusobacterium varium

Bacteroides splanchnicus

Fusobacterium sp.

Eubacterium sp.

Clostridium perfringens

Clostridium ramosum

\section{CULTURE}

The aerobic cultures were performed on horse blood agar (BHIA) and incubated at $37^{\circ} \mathrm{C}$ for 24 hours. The culture for anaerobic bacteria was performed on Columbia agar (BBL) enriched with $10 \%$ horse blood, $5 \mu \mathrm{g} / \mathrm{ml}$ haemin (BDH), and $10 \mu \mathrm{g} / \mathrm{ml}$ vitamin $\mathrm{K}_{1}$ (Merck). The agar plates were incubated in GasPak jars for five days under anaerobic conditions. The subculture of anaerobic bacteria is performed by dividing the agar plate into four and streaking each type of colony on one-quarter of the plate. One colony is streaked on one-quarter of an agar plate for aerobic incubation and on one-quarter of a plate for anaerobic incubation. The anaerobic bacteria that were isolated after 24 hours' incubation were replated into deep agar so that their tolerance to air could be studied as well as the type of medium for their identification according to the method of Holdeman et al. ${ }^{9}$

\section{IMMUNOFLUORESCENCE}

The slides were examined with a Leitz Dialux microscope equipped with a blue filter KP 500. The light source was an Osram lamp Hbo $50 \mathrm{~W} \mathrm{AC}$ with mercury vapour. The slides were read with a $\times 100$ magnification (lens 100/1.20.W Fluorezenz Leitz Wetzler).

The results were graded on a scale from zero to ++++ , according to the fluorescent intensity of the bacterial membrane. If only a slight fluorescence was present, which covered the entire bacterial cell without a strong peripheral component, the reaction was considered to be negative. The positive reactions were those with a fluorescence of +++ to ++++ .

\section{PREPARATION OF CLINICAL MATERIAL}

The test material is first diluted to an opacity of 2 or $3 \mathrm{U}$ on the McFarland scale with physiological water. It is then spread evenly on a glass slide. The slide is air-dried and gently heat-fixed.

A drop of rhodamine pre-stain is placed on the slide, which is then incubated in a moist, dark chamber at room temperature for 15 minutes. The slide is blotted dry with filter paper. Conjugate for either B. fragilis or B. melaninogenicus is placed on the slide, which is returned for an additional 15 to 30 minutes' incubation. The slide is first rinsed with a buffer solution at $\mathrm{pH} 7.2\left(\mathrm{NaCl} 8.5 \mathrm{~g}, \mathrm{Na}_{2} \mathrm{HPO}_{4}\right.$ $2.8 \mathrm{~g}, \mathrm{NaH}_{2} \mathrm{PO}_{4} 0.3 \mathrm{~g}, \mathrm{H}_{2} \mathrm{O} 100 \mathrm{ml}$ ) and then rinsed with distilled water. The slide is allowed to air-dry. A small drop of buffered glycerol ( $\mathrm{pH} \mathrm{7.2)}$ and then a coverslip are added to the smears.

\section{Results}

SPECIFICITY OF FLUORESCENT ANTISERA

The $B$. fragilis and $B$. melaninogenicus antisera exhibited a true specificity for the bacteria of their respective groups (Table 1). The antisera did not react with other bacteria, whether they were aerobic or anaerobic. However, the fragilis antiserum gave a positive fluorescence with Clostridium ramosum spores as well as Staphylococcus aureus and albus. The melaninogenicus antiserum gave a positive fluorescence with Bacteroides bivius.

\section{CULTURE RESULTS COMPARED TO}

IMMUNOFLUORESCENCE

Purulent material was examined and cultured from 119 specimens. Thirty-seven specimens were positive for anaerobic bacteria, many with several species of bacteria. Twenty-three percent of 67 anaerobic strains were $B$. fragilis and $15 \%$ were $B$. melaninogenicus.

In the $B$. fragilis group there were two instances $(1.68 \%)$ where the immunofluorescence was negative 
Table 2 Comparison of results obtained in clinical specimens with immunofluorescence and classic anaerobic culture

\begin{tabular}{|c|c|c|c|c|}
\hline \multirow[t]{3}{*}{ Culture } & \multirow{2}{*}{\multicolumn{2}{|c|}{$\begin{array}{l}\text { B. fragilis } \\
\text { (119 specimens) } \\
\text { Immunofluorescence }\end{array}$}} & \multirow{2}{*}{\multicolumn{2}{|c|}{$\begin{array}{l}\text { B. melaninogentcus } \\
(27 \text { specimens }) \\
\text { immunofluorescence }\end{array}$}} \\
\hline & & & & \\
\hline & Positive & Negative & Positive & Negative \\
\hline Positive & 20 & 2 & 9 & 0 \\
\hline
\end{tabular}

and the culture was positive. Thirteen specimens $(10.92 \%)$ gave a positive immunofluorescence but were negative on culture. Twenty specimens were culture positive, immunofluorescent positive, and 84 specimens were negative for both culture and immunofluorescence (Table 2). There was a correlation between culture and immunofluorescence for 104 clinical samples $(87 \cdot 39 \%)$.

In the $B$. melaninogenicus group, agreement between the culture and immunofluorescence was observed in 22 of the 27 specimens $(81.48 \%)$. No specimen showed disagreement between immunofluorescence and the culture.

\section{Discussion and conclusions}

The first part of the study, which included only strains from our collection, demonstrated the specificity of the $B$. fragilis (group) and $B$. melaninogenicus (group) antisera for anaerobic and aerobic bacteria. The spores of $\mathrm{Cl}$. ramosum and Staph. albus and aureus gave a positive fluorescence with $B$. fragilis antiserum. The morphology of these bacteria is sufficiently well defined for mistakes to be avoided when these microorganisms are examined under the microscope.

$B$. bivius gave a positive fluorescence with the B. melaninogenicus antiserum. B. bivius and Bacteroides disiens, with the exception of some black pigmentation of their colonies, have numerous characteristics in common with $B$. melaninogenicus, for example, biochemical, red fluorescence of the colonies with UV light, and an identical GC percentage of the DNA. ${ }^{10}$ It can be questioned to what extent these two bacterial strains do not belong to the group $B$. melaninogenicus. We were unable to test pure cultures of $B$. disiens with the melaninogenicus antiserum. If this hypothesis is proven, the antigenic relationship between $B$. bivius and $B$. disiens and the group melaninogenicus could be established.

Anaerobic bacterial culture, even if all precautions are taken during transport and with the culture medium, has been known to fail.

In our study, fluorescence showed the presence of $B$. fragilis in 33 samples, while culture was positive in 20 of these cases. B. melaninogenicus was found similarly in five samples out of 27 by fluorescence. We may conclude that this finding does not indicate false-positive reactions since the first part of our study, despite the small number of strains tested, showed that the antisera are very specific for $B$. fragilis and $B$. melaninogenicus group organisms. These reactions could be due to organisms that have died in transport. They would be negative on culture but would be able to give a positive fluorescent reaction. Organisms such as Proteus mirabilis in the culture or the presence of other rapidly growing aerobic bacteria would also explain why the anaerobic cultures were negative. Two false-negative reactions with the antisera were observed for $B$. fragilis. In the first case there was a paucity of bacteria in the initial inoculum. A total of 13 colonies of $\boldsymbol{B}$. fragilis were found on the culture plates. The second case dealt with a $B$. fragilis isolated from a blood culture bottle. Fluorescence on a pure culture of this strain was also negative. It is possible that the serotype of this strain is not included in the $B$. fragilis polyvalent antiserum. The polyvalent antiserum is composed of a group of the major types of strains found in the United States.

The advantages of this immunofluorescence test appear to be considerable. The method is specific and the margin for error is very limited; the test gives a semiquantitative estimate of the number of bacteroides in the clinical specimens in the presence of a mixed flora; and the method is valid for nonviable bacteria. This is particularly important when evaluating cases where the samples have been improperly handled before being cultured. The evaluation is not limited as to type of sample, for example, contamination by a saprophytic flora, nor by the time of procedure. The fluorescent antiserum is polyvalent. The anti-melaninogenicus serum allows the detection of organisms of the group B. melaninogenicus. The anti-fragilis serum allows detection of B. fragilis, Bacteroides ovatus, Bacteroides thetaiotaomicron, Bacteroides distasonis, Bacteroides vulgatus, and Bacteroides uniformis. The method is simple, and the results are obtained rapidly within 45 to 60 minutes.

To conclude, the technique for the detection of $B$. fragilis and $B$. melaninogenicus by immunofluorescence with the Fluoretec $F$ and $M$ kits allows for the use of empirical treatment of bacteroides infections. This is possible in a higher percentage of cases than those that are evaluated by culture alone.

\section{References}

${ }^{1}$ Finegold SM, Sheperd WE, Spaulding EH. Practical Anaerobic Bacteriology Cumitech 5. Washington DC: American Society for Micrøbiology, 1977. 
${ }^{2}$ Gorbach SL, Bartlett JC. Anaerobic infections. $N$ Engl J Med 1974;290:1177, 1237.

3 Holland JW, Hill EO, Altemeier WA. Numbers and types of anaerobic bacteria isolated from clinical specimens since 1960. J Clin Microbiol 1977;5:20.

4 Martin WJ. Isolation and identification of anaerobic bacteria in the clinical laboratory. A 2 years' experience. Mayo Clin Proc 1974;49:301.

${ }^{5}$ Coons AM, Creech HJ, Jones RN, Berliner E. The demonstration of pneumococcal antigen in tissues by the use of fluorescent antibody. J Immunol 1942;45:159.

- Kasper LD, Fildian AP, Tabaochali S. Rapid diagnosis of bacteroides infections by indirect immunofluorescence assay of clinical specimens. Lancet 1979;i:239.

7 Lambe DW. Determination of Bacteroides melaninogenicus serogroups by fluorescent antibody staining. App Microbiol 1974;28:561.
${ }^{8}$ Stanffer LR, Hill EO, Holland JW, Altemeier WA. Indirect fluorescent antibody procedure for the rapid detection and identification of Bacteroides and Fuscbacterium in clinical specimens. J Clin Microbiol 1975; 2:337.

${ }^{9}$ Holdeman LV, Cato EP, Moore WEC, eds. Anaerobe Laboratory Manual. 4th ed. Blacksburg: Anaerobe Laboratory, Virginia Polytechnic Institute and State University, 1977.

${ }^{10}$ Holdeman LV, Johnson JL. Bacteroides disiens sp. nov. and Bacteroides divius sp. nov. from human clinical infections. Int J System Bact 1977;27:337.

Requests for reprints to: Dr M Labbé, Hôpital Universitaire Brugmann, Service du Biologie Clinique, Avenue JJ Crocq 1, 1020 Brussels, Belgium. 